\title{
Le silence éditorial de Gatien Lapointe, ou comment écrire après soi
}

\author{
Jacques Paquin \\ Université du Québec à Trois-Rivières
}

Le parcours éditorial de Gatien Lapointe fait voir une coupure nette entre les œuvres publiées dans les décennies 1950 et 1960 et celles qui paraissent à l'aube des années 1980. Sur le strict plan éditorial, si on exclut les poèmes publiés dans les journaux ou en revue ${ }^{1}$, il s'est déroulé treize années avant que Lapointe ne fasse paraître un recueil. La réflexion que j'entends

\footnotetext{
1 Lapointe a publié dès 1976 des poèmes dans la revue fondée par Yves Boisvert et Bernard Pozier, Atelier de production littéraire de la Mauricie. Ces poèmes ont été ensuite repris dans les publications des années 1980. Rappelons aussi qu'il publie un poème, "Chorégraphie d'un pays », rédigé en 1980 (Québec, album de photographies de Mia et Klaus, Montréal, Libre Expression, p. i-viii).
} 
amorcer peut s'articuler autour de deux questions : 1) Qu'est-ce qui explique d'abord ce long silence? 2) Comment Lapointe a-til pu entreprendre le nouveau volet de son œuvre et à partir de quels motifs? Je propose de suivre quelques pistes convergentes qui pourraient conduire chacune à des éléments de réponse à ce questionnement. En premier lieu, je rattacherai ce silence éditorial à la poétique de Lapointe telle qu'elle se manifeste dans le premier volet de son œuvre. J'élargirai ensuite cette investigation au péritexte, qui dénote une inclination pour l'auto-engendrement. Les grandes figures d'influence (Albert Camus, Paul Éluard) seront aussi convoquées, non pas pour présenter exclusivement un travail qui affiche les réseaux intertextuels présents dans l'œuvre mais bien pour montrer de quelle manière Lapointe a dû négocier sa propre place au sein de la poésie française d'abord, puis de la poésie québécoise. Nous toucherons par la suite au cœur de la problématique de cette étude : le «comment écrire après » de Lapointe découlerait d'une mise à l'épreuve à laquelle s'est soumis le poète avant de publier à nouveau en 1980. De son point de vue, la question se posait plus fondamentalement comme ceci : comment écrire après soi? Enfin, j'établirai des liens entre la poétique deleuzienne et la dernière manière du poète, qui révèle que l'apport de la théorie préside à la mise en place d'une poétique renouvelée.

\section{Une poétique éditoriale}

Le parcours éditorial de Gatien Lapointe se divise en deux volets très différenciés. Après avoir fait paraître cinq recueils entre 1953 et 1967, il ne recommence à publier qu'à partir de 1980, où seront édités quatre recueils qui forment la seconde 
période de son œuvre et que la mort viendra interrompre en 1983. Ce qui résume le mieux le trajet de Lapointe, aussi bien dans le premier que dans le second volet de l'œuvre, c'est la poétique de l'origine ou du commencement. Un simple coup d'œil aux intitulés des publications dévoile ce choix esthétique (Le Temps premier, Le Premier Mot). Dans un entretien qui date de 1981, Lapointe ramenait la trajectoire de son œuvre autour de la question de l'enfance et de l'origine :

Comment redonner une enfance à cet homme et à ce monde ruinés? J'ai écrit il y a longtemps : j'avance vers mon enfance. J'avance vers ce temps premier, je ne recule pas vers ce temps premier. Je dois traverser toute l'épaisseur de l'expérience humaine et celle aussi du langage pour pouvoir rendre réel et habitable ce temps-là. (Smith, 1983, p. 200)

Toute l'entreprise de l'œuvre consiste donc à maintenir un temps mythique où il sera loisible pour le sujet poétique de "prendre demeure à jamais », cette demeure se matérialisant au sein d'un paysage et ayant des affinités avec l'enfance. François Dumont a synthétisé cette poétique qui traverse chacun des recueils:

Depuis le matin «malaisé » qui ouvre son premier poème jusqu'au matin serein qui clôt son dernier recueil, Gatien Lapointe a [...] prioritairement axé son cheminement sur l'isotopie de l'origine. L'itinéraire du poète pourrait se résumer comme suit: vient d'abord la nostalgie de l'enfance dans Jour malaisé et Otages de la joie, puis l'expression du mythe de l'origine dans le Temps premier; la naissance par l'appropriation du sol natal dans l'Ode au Saint-Laurent; l'affrontement entre naissance et mort dans le Premier Mot; la conception du corps comme origine absolue dans Arbre-radar. (p. 77)

Cet aspect déterminant de la poétique du commencement perpétuel se vérifie aussi dans le rapport que Lapointe entretenait avec sa propre écriture. Le poète multiplie les 
ruptures face à sa poétique lorsqu'elle lui apparaît obsolète en regard de nouveaux projets d'écriture. Ainsi, dans l'entretien qu'il accorde à Smith, il pose un jugement très sévère sur les recueils qui ont paru avant les années 1970. Il commente la parution de Jour malaisé en ces termes: "Ce sont là des brouillons de poèmes que j'ai publiés trop tôt. " (Smith, 1983, p. 184) Ensuite, à la question «Pourquoi avez-vous choisi le titre Ode au Saint-Laurent? », Lapointe rétorque : «J'appelais ça d'abord l'homme en marche, et c'était bien ainsi, me semblait-il. Puis, avec les bons conseils de bons amis, ça a pris ce titre horrible. Ça a donné au livre un gentil petit air folklo. Comment faire oublier ce titre maintenant?» (p. 193) Dans une lettre datée du 16 février 1971, Alain Horic, éditeur de la maison de poésie l'Hexagone, avait invité Lapointe à publier l'intégrale de son œuvre au sein de la prestigieuse collection "Rétrospectives", ce à quoi le poète avait répondu favorablement, tout en proposant une nouvelle structure pour le rassemblement de ses écrits. L'examen des archives personnelles du poète m'a permis de trouver ce qui s'apparente au brouillon de la préface qu'il comptait sans doute soumettre en même temps que la rétrospective. Mais le projet avorta parce que Lapointe avait insisté pour apporter des corrections majeures aux éditions originales.

Les rapports qu'entretient Lapointe avec ses écrits antérieurs se révèlent donc problématiques mais il faut comprendre ce retournement comme l'expression d'une fidélité à soi-même qui a des racines profondes. Cette rupture, loin de constituer une tabula rasa de sa poétique, est une manière de commencer ou, si l'on veut, de relancer à chaque publication un rythme, une phrase qui ramène à l'origine. Par exemple, on peut considérer Arbre-radar comme une nouvelle version du chant 
du monde amorcé par l'Ode au Saint-Laurent, mais dont l'objet se serait déplacé, passant de la célébration du pays à l'exaltation du corps. Comme l'écrit encore Dumont, « [s]i par la suite son style se transforme, c'est surtout pour "coller" davantage au corps, qui devient vraiment, pour lui, source de l'écriture, et aussi pour manifester le recommencement de l'écriture dans la phrase même » (p. 78).

Cette renaissance perpétuelle qu'ambitionne Lapointe l'amène à proclamer l'instantané comme une valeur absolue, de sorte que le métadiscours mis de l'avant par le poète, la teneur de ses commentaires sur son œuvre donnent au lecteur l'impression que la série des recueils se réduit à une suite de lignes brisées, où chaque parution n'aurait pas visé d'autre objectif que celui de corriger les erreurs du précédent. Lapointe est intransigeant avec lui-même et peu de ses écrits trouvent grâce à ses yeux, comme en témoigne l'entretien avec Smith au cours duquel le poète, s'il ne va pas jusqu'à renier l'Ode au Saint-Laurent, ne se gêne pas pour condamner l'intitulé en raison de ses connotations nationalistes. Certes, cet entretien se déroule à l'aube des années 1980, à une époque où la ferveur nationaliste s'est progressivement essoufflée après l'échec du Référendum de 1980 visant à déterminer si le Québec allait opter pour une souveraineté-association avec le Canada. Cette conjoncture politique demeure toutefois insuffisante pour expliquer cette propension de l'auteur à juger aussi sévèrement ses écrits passés.

Rappelons le projet de rétrospective prévu à l'Hexagone, qui est demeuré lettre morte. Lapointe est incapable de se relire sans apporter un jugement généralement dépréciatif sur son œuvre. Par exemple, la publication successive de recueils en auto-éditions, Jour malaisé (1953) et Otages de la joie (1955), 
traduit sans aucun doute le refus de se soumettre à l'opinion d'un éditeur et d'être le maître d'œuvre de ses propres recueils ${ }^{2}$; mais ce choix semble exprimer aussi un sentiment d'urgence, qui ne quittera jamais le poète jusqu'à la fin de sa vie. Bien que Lapointe n'ait apparemment pas reconnu publiquement l'influence de Saint-Denys Garneau sur sa poésie, on ne peut manquer de lier la poétique de l'auteur du Premier mot au fameux liminaire garnélien : « Je ne suis pas bien du tout assis sur cette chaise / Et mon pire malaise est un fauteuil où on reste / Immanquablement je m'endors et j'y meurs » (1996, p. 33). À l'expression poétique «je nais dans chaque mot» (Lapointe, 2000, p.44), que nous pouvons lire dans "J'appartiens à la terre», qui précède l'Ode au Saint-Laurent, correspond le désir chez le poète de se délester d'une pratique qu'il ne juge plus suffisamment fidèle à la poétique de l'ici et maintenant.

\section{Les marques de l'auto-engendrement}

Cette posture rapproche le sujet lapointinien du fantasme d'auto-engendrement, dont je tire la définition d'un descriptif du colloque organisé par Frédérique Bernier, qui s'est tenu à l'Acfas, à l'Université d'Ottawa, en 2009: "Désignant un processus par lequel un sujet tente de se donner à lui-même ses propres fondements, sa propre origine, la notion d'autoengendrement renvoie d'abord à un projet littéraire moderne conçu, dès les romantiques allemands, en terme d'autopoïesie, de production de soi. »

\footnotetext{
${ }^{2}$ Lapointe s'appuie sans doute à l'époque sur l'expertise qu'il a acquise lors de ses études à l'École des arts graphiques de Montréal, au début des années 1950, où il s'est initié à la confection matérielle d'un ouvrage littéraire.
} 
Cet auto-engendrement est palpable non seulement dans une poétique où le sujet affirme haut et fort qu'il naît dans chaque mot qu'il prononce, mais aussi dans le péritexte de l'œuvre. Ainsi, le second recueil, Otages de la joie, s'ouvre sur une épigraphe de son auteur, identifiée par ses initiales G. L. et elle est suivie d'un discours préfaciel auctorial où le recommencement est conçu comme un mot d'ordre auquel le poète ne saurait échapper, sous peine de se trahir lui-même : « Le poète doit tout recommencer, si l'ange n'était pas attentif, à partir de cette humilité consciente de n'habiter un royaume grave et cher que si d'abord ce royaume l'affectionne et puis l'invite tout bonnement. » (2001, p.95) Les deux parties du recueil J'appartiens à la terre, suivi de Ode au Saint-Laurent, sont chacune précédées d'une épigraphe de Lapointe et, dans Le Premier Mot, c'est encore un vers du poète qui chapeaute l'ensemble : «Écrire, c'est encore espérer ${ }^{3}$ » (p. 97).

Comme on peut s'en douter, Lapointe n'était pas pour autant imperméable aux influences littéraires. J'ai dénombré quatre figures qui ont influencé à divers degrés la poétique de Lapointe: Éluard, Rimbaud, Nelligan et, dans une moindre mesure, Camus. Le poète lui-même n'a pas manqué d'attirer l'attention sur ces figures, que ce soit dans l'espace des exergues, dans des entrevues ou même au sein de sa poésie. Pour les besoins de la problématique de ce collectif, j'écarte délibérément les noms de Rimbaud et de Nelligan parce qu'ils ne sont pas des cas de figure problématiques du «comment écrire », à la différence des autres.

\footnotetext{
${ }^{3}$ Hormis l'ajout d'une virgule, cette épigraphe reproduit textuellement un passage de la préface (p. 98).
} 
L'œuvre de Camus a exercé une influence notable davantage chez le premier Lapointe. On en trouve des traces dans l'essai "Le pari de ne pas mourir », qui sert de préface au Premier Mot (1967). On peut y lire en filigrane l'interrogation qui ouvre L'Homme révolté : «Qu'est-ce qu'un homme révolté? Un homme qui dit non. » (Camus, p. 25) Lapointe écrit à son tour : «La poésie c'est d'abord pour moi un homme condamné à mourir et qui dit NON. » (2000, p. 97) Si l'art et la poésie sont pour lui la solution à l'absurdité de la vie, le poète ne les considère pas moins comme une manifestation parmi tant d'autres. Pour Lapointe, la beauté, « ce n'est pas un poème, ni une page de musique, ni un tableau, c'est la vie elle-même. » (2000, p. 104) Cette préséance donnée à la vie, qui se traduit chez lui par une confiance absolue en l'habitation du corps, plus encore que dans le vers ou même la parole proférée, rejoint aussi le premier Camus, plus lyrique, celui de Noces, et dont l'exemplaire de la bibliothèque personnelle de Lapointe donne un aperçu de cette influence, notamment dans les passages soulignés, comme ceux-ci : "Car le corps ignore l'espoir. il ne connaît que les coups de son sang. » (Camus, p. 54); et plus loin : "L'immortalité de l'âme, il est vrai, préoccupe beaucoup de bons esprits. Mais c'est qu'ils refusent, avant que d'en avoir épuisé la sève, la seule vérité qui leur soit donnée et qui est le corps. » (p.55) Ce pari de ne pas mourir énonce un constat assez sombre: "Chaque instant nous répète que toute existence est un échec. » (Lapointe, 2000, p. 97); mais il part aussi d'un sentiment d'impuissance face au langage, toujours suspect aux yeux du poète lorsqu'il n'est pas l'expression de la vie elle-même. En somme, Lapointe est en quête du mot originel, en proie à une pensée cratylique où le signifiant s'avérerait l'expression directe, presque sans médiation, de la 
vie qui bat. Ce rêve mimétique, on en trouve des échos lointains dans Noces, dont Lapointe a souligné ce passage :

Comme le galet verni par les marées, j'étais poli par le vent, usé jusqu'à l'âme. J'étais un peu de cette force selon laquelle je flottais, puis beaucoup, puis elle enfin, confondant les battements de mon sang et les grands coups sonores de ce cœur partout présent de la nature. Le vent me façonnait à l'image de l'ardente nudité qui m'entourait. (p. 25)

L'influence de Camus, toutefois, ne sera pas aussi persistante que celles de Rimbaud et d'Éluard ${ }^{4}$, qui a exercé un ascendant beaucoup plus déterminant dans la poétique lapointienne. Lapointe fréquente déjà l'œuvre d'Éluard lors de la parution de son premier recueil. En 1956, il a déposé un mémoire de maîtrise consacré au poète surréaliste intitulé "L'expérience intérieure de Paul Éluard». Il profite, au cours de la même année, d'une bourse de la Société royale du Canada pour entreprendre des études de doctorat à la Sorbonne. Il y soumet un sujet de thèse, "La lumière chez Paul Éluard », mais il ne mènera pas à terme ses recherches. Ce corpus d'élection est disséminé dans l'œuvre du poète québécois à travers un échantillonnage dont je ne donnerai que quelques exemples :

Et tu es encore là sous mes paupières (Lapointe, 2001, p. 295) Jadis dans tes yeux ta chevelure J'ai voulu rassembler des étoiles mortes Pour faire un feu au pied du ciel (p. 636)

\footnotetext{
4 Toutefois, elle n'est pas éteinte pour autant puisque Lapointe précise, dans son entretien avec Smith : "Je reviens souvent à Noces de Camus.» (1983, p. 182)

5 «Aux coquilles du rêve», tiré de Jour malaisé (1953). Ce vers rappelle le premier vers du poème «L'amoureuse » (Éluard, 1966, p. 56).

6 On reconnaît en filigrane les vers de « Pour vivre ici » : « Je fis un feu, l'azur m'ayant abandonné / Un feu pour être son ami / Un feu pour m'introduire dans la nuit d'hiver, / Un feu pour vivre mieux. » (Éluard, 1947, p. 77).
} 
L'avant-dernier poème d'Otages de la joie rappelle indéniablement le poème "Liberté »: «Et tu retrouveras la liberté première de ton enfance » (p. 126), tandis que le dernier poème du même recueil (p. 127) place ces vers extraits d'«Au bien » en épigraphe :

Nous avons pénétré le feu

Il faut qu'il nous soit la santé

Nous nous levons comme les blés

Et nous ensemençons l'amour (Éluard, 1968, p. 314)

Enfin, le poème liminaire du Temps premier établit de nouveau cette parenté littéraire avec "Je nais dans tout ce que je nomme » (p. 127), écho manifeste du célébrissime «Liberté » d'Éluard. Dumont, de son côté, n'a pas manqué de relever, à propos du même recueil, des ressemblances jusque dans le phrasé (voir 1989, p. 27).

Il n'en demeure pas moins que la publication du Temps premier coïncide avec une rupture, du moins partielle, avec l'auteur de Capitale de la douleur. Alors que Lapointe était en terre française, la rédaction du Temps premier a été pour lui l'occasion de prendre ses distances simultanément vis-à-vis de son objet d'étude et de son modèle littéraire. Le poète a témoigné du sentiment d'exil très fort qu'il a éprouvé à l'époque et qui est à l'origine de ce recueil :

Suffoquant un jour, j'ai pris conscience de ce qui m'arrivait : je n'étais pas de ce pays-là, je n'arriverais jamais à prendre racine sur une autre terre que la mienne [...].

Et intérieurement, sur un autre pied, avec une autre attitude, je commençais mon deuxième voyage en France. [...]. Au lieu de toujours essayer de traduire, je commençais à affirmer mon propre langage, et d'abord, contre celui d'Éluard qui m'oppressait tout en me fascinant. Je surgissais dans un temps premier, dans mon propre temps et dans mon propre espace. 
Le Québec, de loin, à ce moment-là, devenait ma terra nova. (Smith, p. 188)

Ainsi, le titre du recueil, quoique suggéré par un tiers, est luimême l'expression d'une rupture avec un certain type de filiation française. Toutefois, Éluard ne disparaîtra jamais totalement des figures tutélaires du poète, puisque un chapitre d'Arbre-radar lui réserve une citation : «Le monde est sur la table des métamorphoses ${ }^{7}$ » (Lapointe, 1980, p. 45). Comme on peut le supputer, la référence au poète français se manifeste sur d'autres bases que la célébration ou la lumière. Le choix même de cette épigraphe, me semble-t-il, est significatif de ce déplacement : Éluard n'est plus l'emblème de la plénitude ou de l'immuable beauté, le passage prélevé dans ce nouveau volet poétique dit à son tour, méta-poétiquement en somme, le changement de perspective et d'horizon envisagé depuis le recueil de $1967^{\circ}$. C'est ce qui nous amène aux causes qui ont pu motiver cet intervalle de treize années de silence éditorial. Je proposerai deux pistes qui, d'ailleurs, ne s'excluent pas l'une l'autre.

\section{Silence éditorial}

D’abord, ce hiatus pourrait s'expliquer par la décision de Lapointe de briguer un poste de professeur de création et de littérature à l'Université du Québec à Trois-Rivières, qui ouvre ses portes en 1969. Non seulement doit-il faire face à de

\footnotetext{
${ }^{7}$ Ce vers est extrait du poème intitulé « Une image revient à qui l'a mise au monde », dans Léda, Éditions Mermod, Lausanne, 1949 (Éluard, 1968, p. 264). ${ }^{8}$ Bien que je m'en tienne à la date d'édition des recueils, il n'est pas inutile de préciser que la date de rédaction du Premier Mot précède de trois années celle de sa publication, comme on peut le lire au bas du dernier poème : « Saint-Jean-surRichelieu / automne 1963 — automne 1954 » (Lapointe, 2000, p. 163).
} 
nouvelles responsabilités, mais, au cours de ses deux premières années d'embauche, il travaille à la fondation d'une maison d'édition de poésie, Les Écrits des Forges, qu'il dirige jusqu'à sa mort en 1983.

L'autre raison tient à la pression que lui fait subir sa propre renommée. En effet, Lapointe a remporté des prix pour Le Temps premier, en France, ainsi qu'au Québec pour L'Ode au Saint-Laurent, en 1963, et Le Premier Mot en 1967. Il a même cumulé plusieurs prix pour un même ouvrage. On peut donc présumer que la barre était haute à ses yeux et que, ayant consacré toutes ses énergies à son enseignement et à sa maison d'édition, ayant aussi vécu pendant ce temps sur le capital accumulé de cette renommée de poète, il a dû, à un certain moment, se demander s'il pourrait encore continuer à défendre son titre de poète national. Quand a lieu la Nuit de la poésie, au théâtre Gésu, en 1970, Lapointe, alors âgé de 38 ans, lâche cette réflexion alors qu'il pénètre dans l'enceinte du théâtre : "ça ne nous rajeunit pas» (Labrecque, 1970). Il approche de la quarantaine et mesure sans doute avec un peu d'angoisse les changements importants qui ont marqué le paysage poétique québécois depuis 1967. Lapointe est ainsi vraisemblablement confronté à un doute: sera-t-il en mesure de se renouveler? Sera-t-il à la hauteur de son propre renom?

D'une part, il devait savourer cette reconnaissance de poète, qui en partie lui a ouvert les portes de l'université et lui conférait un ascendant sur ses collègues, ses étudiants et au sein de sa maison d'édition. D'autre part, comme on l'a vu, il ne pouvait se résoudre à répéter purement et simplement ce qu'il avait déjà fait, au risque d'être inconséquent par rapport à sa poétique du commencement. D’ailleurs, la période littéraire ne 
s'y prêtait plus guère. Le marché poétique était beaucoup plus concurrentiel et les projecteurs étaient désormais braqués sur la génération montante. En revanche, on peut envisager de manière positive cette angoisse de l'influence, concept forgé par Bloom et selon lequel tout poète est confronté à l'obligation d'originalité en dépit de l'influence que peuvent exercer sur lui ses précurseurs. Lapointe, à n'en pas douter, éprouvait cette angoisse et prenait très au sérieux les conseils qu'on lui prodiguait ou les avis qu'on énonçait sur sa poésie. Les choix d'intitulé pour Le Temps premier et Ode au Saint-Laurent lui ont été proposés par des tiers. Il faudrait aussi tenir compte des échanges d'égal à égal qu'il établissait avec ses étudiants. Leurs avis lui étaient précieux, comme en fait foi un récit de Bernard Pozier, l'étudiant qui lui a succédé à la direction littéraire des Écrits des Forges :

Mes mains tremblaient et, lorsqu'il sollicita mon opinion, ma voix tremblait tout autant et j'avais bien hâte de voir les autres s'amener. J'avais dû ne pas trop dire de bêtises puisque, par la suite, j'ai eu l'honneur de lire presque toutes ses ébauches de textes, d'en discuter avec lui, parfois de lui suggérer quelques petites choses et, à ma grande surprise, souvent de le rassurer, car lui aussi connaissait le vertige et la peur de tous les créateurs. (p. 31)

Par ailleurs, Lapointe avait pour collègue et ami Armand Guilmette, qui s'intéressait de près à la théorie deleuzienne, qu'il enseignait dans ses séminaires de maîtrise. Lapointe, qui était réfractaire à une poésie abstraite ou hermétique, reconnaissait en Guilmette un théoricien ${ }^{9}$, alors que lui-même

\footnotetext{
${ }^{9}$ Guilmette a publié une série d'études inspirées de la schizo-analyse (Gilles Deleuze et la modernité) qui témoignent de son intérêt pour cette approche au cours des années où il a côtoyé Lapointe. L'ouvrage se clôt sur un article consacré à la poétique lapointinienne.
} 
se considérait plutôt comme un instinctif. Dans sa volonté de rompre avec une poétique qu'il jugeait obsolète, le poète a sans doute aperçu, dans la théorie de la schizo-analyse deleuzienne, une confirmation de la nouvelle manière de sa poésie. On trouve dans ses archives des notes sur Deleuze qui laissent à penser qu'il a pu soumettre sa propre poétique à cette lecture, en particulier à partir de l'ouvrage Rhizome, co-signé par Deleuze et Guattari. Cette nouvelle image, qui vient donner une autre valeur à l'arborescence traditionnelle pratiquée par le poète jusque-là, lui permet d'inscrire dans sa démarche des «lignes de forces» - le terme est deleuzien - qui vont désormais prendre leur source dans le corps et se développer non plus verticalement, mais horizontalement, par ramifications sonores favorisées par l'importance que prennent dans Arbre-radar les jeux du langage qui mettent en scène une poétique « accomplissant l'antique projet de l'arbre » (Lapointe, 1980, p. 56). La note manuscrite de Lapointe que livre Pozier dans son ouvrage signale l'influence déterminante des écrits deleuziens:

L'arbre-enfant, c'est l'arbre au fonctionnement tout croche, rhizomatique, circulaire, spiralique, qui coupe à mesure la tête du discursif, qui se déterritorialise constamment, qui fait des sauts à côté, dirait un grand petit livre RHIZOME, que j'ai lu en décembre 1976 [...]. (Pozier, p. 140)

La poétique du commencement est toujours présente, mais elle a remplacé l'habitation du pays par celle du corps, devenu table à sensations (rappelons-nous le vers d'Éluard cité plus haut), qui donne lieu à une poésie dans laquelle les réseaux phoniques, à travers l'isotopie de l'énergie, électrique entre autres, permettent d'atteindre le « jouir », vocable récurrent de cette seconde manière. Toutefois, bien que cette seule et unique 
contribution au catalogue de l'Hexagone ait suscité beaucoup d'intérêts et des critiques - positives, pour la plupart - , il existe une marge entre le concert d'éloges qui a accueilli les recueils des années 1960 et la réception critique d'Arbre-radar. Lapointe nourrissait des attentes si élevées que Pozier suppose que son maître et ami fut déçu par la réception critique de l'ouvrage, qui ouvrait une seconde manière dans sa poésie:

[...] Gatien n'en parlait pas beaucoup, mais il fut sans doute attient par un certain manque de cette reconnaissance et de cette gloire que ses précédents recueils lui avaient procurées à foison. Quelques propos expéditifs, pontifiés du haut de quelques plaquettes, ne lui semblaient certes pas à la hauteur de son labeur. (p. 27)

Les recueils publiés ultérieurement maintiennent la même veine, mais empruntent alors une forme plus artisanale : ce sont des plaquettes qui semblent indiquer soit un tarissement de la source créatrice, soit un choix délibéré de publier des ouvrages d'art à tirage limité. À tout le moins, peu importe que le souffle du poète s'amenuise, ce dernier ne remettra plus en question les œuvres publiées subséquemment.

L'hypothèse développée au cours de ces pages sur l'angoisse provoquée chez le poète à l'idée d'écrire après soi a pris deux formes. La première a trait à la filiation littéraire, où ce « comment écrire » prend chez Lapointe la forme d'un écrire après soi, c'est-à-dire, en se délestant d'influences françaises trop marquées, comme celle d'Éluard. Cette prise de conscience coïncide avec un retour au Québec, où le poète redécouvre son appartenance québécoise et terrienne, à l'origine de la publication de l'Ode au Saint-Laurent ${ }^{10}$. En 1967, Le Premier Mot

10 On n'oubliera pas, néanmoins, que ce poème a été écrit en France. Bien qu'il ait connu quatre éditions, l'indication du lieu d'écriture a toujours été 
semble proposer une esthétique renouvelée, moins unitaire que celle de l'Ode sur le plan formel à tout le moins. La discontinuité typographique des vers reflète la précarité du sujet lapointinien, qui clôt le premier cycle des recueils. La palette du temps a exercé une influence notable sur l'œuvre, à un double titre. Comme on l'a vu, Lapointe relègue parmi les textes d'un passé révolu tout ce qui précède Le Premier Mot comme si, justement à ses yeux, cette pratique était littéralement dépassée. D’autre part, le temps qui fuit lui fait éprouver d'autant plus la crainte - que partage tout artiste - de n'être plus de son temps. Par conséquent, il a pu appréhender qu'il doive surtout sa reconnaissance littéraire à l'Ode, devenue d'ores et déjà un classique de la poésie québécoise et de la poésie dite du pays en particulier, à laquelle il refusait pourtant de s'identifier. Son pari de ne pas mourir, qui imprègne encore fortement le recueil de 1967 et qui le rattache toujours à la thématique du pays, revêt une signification qui met aussi en jeu sa propre pratique esthétique puisque Lapointe voulait rester un poète de son temps. Le défi pour lui aura donc consisté à recommencer, tout en se refusant à se répéter, par souci de rester à l'avant-garde de la poésie québécoise. D'où cette longue période de latence où le poète a dû reconnaître qu'il n'était plus aussi jeune, tout en travaillant à garder intactes ses capacités d'étonnement, en maintenant un équilibre fragile entre un « écrire après » (les autres) et un « écrire (d') après » soi.

maintenue en fin de poème: «Paris, janvier 1961 ». Il est issu d'un choc lorsque le jeune Lapointe revient au pays par bateau pour rendre visite à ses proches et qu'il découvre le fleuve à son embouchure. 


\section{Bibliographie}

Bloom, Harold. (1973), The Anxiety of Influence. A Theory of Poetry, Londres, Oxford University Press.

CAmus, Albert. (1968 [1951]), L'Homme révolté, Paris, Gallimard, coll. « Idées ».

—. (1959 [1938]), Noces suivi de L'été, Paris, Gallimard, coll. «Folio ».

DELEUZE, Gilles et Félix GUATTARI. (1976), Rhizome. Introduction, Paris, Minuit.

Dumont, FrançoIs. (1989), L'Éclat de l'origine. La poésie de Gatien Lapointe, Montréal, L'Hexagone.

ÉLuARD, Paul. (1968 [1949]), CEuvres complètes, vol. 2 (éd. critique de Lucien Scheler et Marcelle Dumas), Paris, Gallimard, coll. « Bibliothèque de la Pléiade».

—. (1966 [1926]), Capitale de la douleur suivi de L'amour la poésie, Paris, Gallimard, coll. « Poésie ».

—. (1947), Le Livre ouvert : 1938-1944, Paris, Gallimard.

GuILMETTE, Armand. (1984), Gilles Deleuze et la modernité, Trois-Rivières, Les Éditions du Zéphyr.

LABRECQUE, Jean-Claude et Jean-Pierre MASSE. (1970), La Nuit de la poésie. 27 mars 1970, Montréal, Office national du film, $111 \mathrm{~min} .11 \mathrm{sec}$.

LAPOINTE, Gatien. (2001), Le Temps premier Uour malaisé [1953], Otages de la joie [1955], Le temps premier [1962]), Trois-Rivières, Écrits des Forges. 
-. (2000), Ode au Saint-Laurent (J'appartiens à la terre [1961], Ode au Saint-Laurent [1963], Le Premier Mot [1967], Chorégraphie d'un pays [1980]), Trois-Rivières, Écrits des Forges.

—. (1980), Arbre-radar, Montréal, l'Hexagone.

-. (1956), «L'expérience intérieure de Paul Éluard», Université de Montréal, maîtrise ès arts, Montréal, 75 f.

PozIER, Bernard. (1987), Gatien Lapointe. L'homme en marche, Trois-Rivières, Écrits des Forges [co-éditeurs : La Table rase (France) et Schena (Italie)].

SAINT-DEnys GARnEAU, Hector de. (1996 [1937]), Regards et jeux dans l'espace, Montréal, Bibliothèque québécoise.

SMITH, Donald. (1983), « Gatien Lapointe : du pays retrouvé à la primauté du corps", dans L'Écrivain devant son œuvre, Montréal, Québec/Amérique, p. 177-203.

\section{Résumé}

Cet article aborde le parcours éditorial et poétique de Gatien Lapointe à la lumière de deux aspects qui ont exercé une influence déterminante sur sa poésie. En premier lieu, l'auteur soupèse l'importance de certaines filiations littéraires (Albert Camus, Paul Éluard) ou théoriques (Gilles Deleuze) ainsi que des ruptures qu'elles provoquent dans la manière du poète, soucieux de se délester de ces influences. En contrepoint, cette étude s'interroge sur les raisons qui ont pu motiver un silence éditorial qui a duré treize années entre la publication du recueil Le Premier Mot (1967) et celle d'Arbre-radar (1980). 


\begin{abstract}
This article discusses the editorial and poetic itinerary of Gatien Lapointe in light of two aspects that have exerted a determining influence on his poetry. First, the author weighs the importance of certain literary (Albert Camus, Paul Éluard) or theoretical (Gilles Deleuze) filiations as well as the conflicts they provoke in the style of the poet eager to jettison these influences. In counterpoint, the article then examines the possible reasons for the thirteen-year editorial silence between the publication of the collection Le Premier Mot (1967) and that of Arbre-radar (1980).
\end{abstract}

